\title{
Inducción Secretoria de IgG, con toxina de Clostridium perfringens Tipo A para la reducción de mortalidad por enterotoxemia en crías de Alpacas
}

\section{Secretory induction of IgG, toxin of Clostridium perfringens type A for the reduction of mortality due to enterotoxemia in offspring of Alpacas}

\author{
Pezo-Carreón Danilo $^{*}$, Alarcón-Bayona Virgilio ${ }^{2}$, Franco-Febres Francisco ${ }^{1}$, Pacheco-Curie Joel ${ }^{1}$
}

\begin{tabular}{l}
\hline DatoS del Artículo \\
\hline Universidad Nacional Mayor de San \\
Marcos. Facultad de Medicina Veterina- \\
ria. Instituto Veterinario de Investigacio- \\
nes Tropicales y de Altura-Marangani. \\
Calle Lima 106 Marangani. Cusco, Perú. \\
Canchis - Cusco, Perú. \\
febranco90@hotmail.com \\
jpachecoc@ unmsm.edu.com \\
2 Universidad Nacional de San Antonio \\
Abad del Cusco. Centro de Investigación \\
en Camelidos Sudamericanos. La Raya. \\
Av. de la Cultura, Nro. 733, Cusco, Perú. \\
Telf. +51.84.604100, 51.84.604160. \\
Fax +51.84.238156. \\
Vialba2106@ @otmail.com \\
*Direción de contacto: \\
Universidad Nacional Mayor de San \\
Marcos. Facultad de Medicina Veterinaria \\
Instituto Veterinario de Investigaciones \\
Tropicales y de Altura-Marangani. \\
Calle Lima 106 Marangani Cusco Perú \\
Canchis - Cusco - Perú \\
Danilo Pezo-Carreón \\
E-mail address: \\
dpezo71@ hotmail.com \\
\hline Palabras clave: \\
\hline Alpacas, \\
Crías, \\
Clostridium perfringens, \\
enterotoxemia, \\
toxina. \\
\hline \\
\hline
\end{tabular}

J. Selva Andina Anim Sci. 2018; 5(2):56-64.

\section{Historial del artículo.}

Recibido octubre, 2017.

Devuelto marzo 2018.

Aceptado agosto, 2018.

Disponible en línea, octubre, 2018.

\begin{tabular}{l}
\multicolumn{1}{c}{ Editado por: } \\
Selva Andina \\
Research Society \\
\hline Key words: \\
Alpacas, \\
Crias, \\
$\begin{array}{l}\text { Clostridium perfringens, } \\
\text { enterotoxemia, } \\
\text { toxin. }\end{array}$ \\
\hline
\end{tabular}

\section{Resumen}

En el IVITA Marangani FMV-UNMSM, con el objetivo de producir inducción secretoria de IgG, con toxina de Clostridium perfringens Tipo A, para reducir la mortalidad por enterotoxemia en crías de alpacas. Se preparó un toxoide a partir de muestras de intestino de crías de alpacas con enterotoxemia positivos a $C$. perfringens tipo A., fueron analizadas empleando medios de cultivo diferenciales en anaerobiosis, la morfología microscópica mostro bacilos Gram (+) cortos. Bioquímicamente se caracterizó lecitinasa + , catalasa -, hidrólisis gelatina,$+ \mathrm{H}_{2} \mathrm{~S}+$, fermentación glucosa + , lipasa -, indol -, crecimiento aerobio -, urea -, nitratos +, movilidad -, esculina - y gas +. Se administró $2 \mathrm{~mL}$ de toxoide a madres preñadas al noveno y décimo mes de gestación y a crías $2 \mathrm{~mL}$ del toxoide a los 10 días de nacidas distribuidos en 4 grupos: 1) madres-crías vacunadas, 2) madres no vacunadas-crías vacunadas, 3) madres vacunadas-crías no vacunadas y 4) madres y sus crías no vacunar. No se presentaron casos de mortalidad por enterotoxemia, el porcentaje de morbilidad fue: $15 \%, 15 \%, 25 \%$ y $30 \%$ para los grupos $1,2,3$, y 4 respectivamente. Los resultados confirmaron que la administración del toxoide preparado a base de cepas de Clostridium perfringens tipo A indujo mayor protección en los grupos 1 y 2 que en los grupos 3 y 4 . Se sugiere vacunación masal a hembras preñadas y crías del hato de alpacas de acuerdo a lo realizado en el grupo 1 . Se concluye que el toxoide induce la protección contra enterotoxemia.

(C) 2018. Journal of the Selva Andina Animal Science. Bolivia. Todos los derechos reservados.

\section{Abstract}

In the IVITA Marangani FMV-UNMSM, with the aim of producing secretory induction of IgG, toxin of Clostridium perfringens type A, to reduce mortality due to enterotoxemia in offspring of alpacas. Prepared a toxoid from young alpacas intestine samples with positive enterotoxemia to $C$. perfringens type a. were analyzed using culture media spreads in anaerobic, microscopic morphology showed bacilli Gram (+) short. Biochemically characterized lecithinase + , hydrolysis, catalase - gelatin,$+ \mathrm{H}_{2} \mathrm{~S}+$, glucose fermentation + , lipase - indole-, aerobic growth-, urea-, nitrate + , mobility-, esculin - and gas + . Was administered $2 \mathrm{~mL}$ of toxoid to mothers pregnant for the ninth and tenth month of gestation and young $2 \mathrm{~mL}$ of toxoid 10 days after born divided into 4 groups: 1) vaccinated mother- young, 2) mothers not vaccinated young vaccinated, 3) vaccinated mothers-unvaccinated young, and 4) mother s and their offspring do not vaccinate. There were no cases of enterotoxemia mortality, morbidity was: $15 \%, 15 \%, 25 \%$ and $30 \%$ for groups 1, 2, 3, and 4 respectively. The results confirmed that the administration of toxoid prepared from strains of Clostridium perfringens type $\mathrm{A}$ induced greater protection in groups 1 and 2 that in groups 3 and 4. Mass vaccination is suggested to pregnant females and young from the herd of alpacas according to what has been done in Group 1. It is concluded that toxoid induces protection against enterotoxemia. 


\section{Introducción}

La enterotoxemia es una enfermedad que genera elevadas tasas de morbi-mortalidad neonatal, ocasionando grandes pérdidas a productores alpaqueros del país. El control de la enterotoxemia se basa principalmente en la higiene, manejo de los animales, que en escasas ocasiones, se realiza la vacunación. ${ }^{1-3}$ En el mercado nacional, se dispone de una vacuna a base de anacultivo del agente, Clostridium perfringens, formalizadas y adsorbidas en hidróxido de aluminio, la que genera una respuesta inmune sistémica, inicialmente la vacuna estuvo conformada por cepas aisladas de casos de enterotoxemia en $80 \%$ de ovinos (tipos A, B, C y D), $20 \%$ de una cepa Tipo A (Cepa Moro) aislada de casos similares en alpacas. ${ }^{4}$

A partir de 2003, las cepas ovinas fueron remplazadas progresivamente por cepas patogénicas aisladas de alpacas. En el 2005, 80\% fueron cepas correspondientes a los tipos $\mathrm{A}, \beta 2$ y $\mathrm{C}$, manteniendo el $20 \%$ restante cepas tipos B y D de origen ovino ${ }^{5}$, la presencia de $C$. perfringens genotipo A subtipo $\beta 2$ toxigénico aislados de casos de enterotoxemia de alpacas, evidencian la posible participación de esta toxina en la etiopatogénesis de la enfermedad, como los principales factores de virulencia asociados en la enterotoxemia de las crías de alpacas. ${ }^{6}$

En este sentido, se ha demostrado la eficacia de inmunógenos en otras especies animales derivados de bacterias patógenas intestinales, brindando resultados promisorios. Estudios recientes señalan la evidencia del efecto in vitro de los antígenos clostridiales, sobre la respuesta inmune, más aún determinaron que los derivados de $C$. perfringens son los más potentes estimulantes, dentro del género Clostridium. $^{7}$ Por otro lado, muchas enfermedades son manejadas a través de la estimulación de una res 57 puesta intestinal eficaz, y actualmente se están continuando estudios de manejo de enfermedades causadas por toxinas, a través del tratamiento oral, como es el caso de los estudios para la modulación de la respuesta frente a las toxinas de Clostridium teta$n i$ en roedores ${ }^{8}$ y en crías de alpacas. ${ }^{9}$

La habilidad protectiva del anacultivo en la enterotoxemia de las alpacas se ha descrito desde los estudios iniciales de la enfermedad en el Perú ${ }^{10}$ y en Chile. ${ }^{11}$ Este tipo de vacuna es muy utilizada en programas de control de la enfermedad en países que han comenzado con la crianza intensiva de alpacas en el resto del mundo. ${ }^{12}$ Los toxoides son excelentes biológicos, ampliamente usados en la protección contra el complejo enterotoxigénico en explotaciones ovejeras de muchos países, incluyendo el Perú. ${ }^{13}$ En alpacas la enfermedad afecta a las crías entre la segunda y tercera semana de edad ${ }^{14}$, periodo crítico que debe ser protegido por los anticuerpos maternales de la madre vacunada antes del parto. ${ }^{15}$

La vacunación exclusiva de crías también reduce sustancialmente los índices de mortalidad neonatal. Sin embargo, es posible que la aparente protección contra la enfermedad obtenida al vacunar a las crías sea debido a la presencia de anticuerpos maternales residuales provenientes de madres vacunadas el año anterior $^{16}$ o de anticuerpos provenientes de una campaña anterior con elevada mortalidad por enterotoxemia, es de allí que surge el concepto, de la denominada ciclicidad de los brotes enterotoxigénicos en las explotaciones alpaqueras, la ciclicidad, puede explicarse por la presencia de protección por anticuerpos en el hato después de brotes naturales de la enfermedad. ${ }^{4}$ 
Los animales sobrevivientes de brotes epizoóticos mantendrían niveles de anticuerpos en cantidades suficientes para proteger al rebaño por un determinado número de años. La disminución progresiva de la cantidad y calidad de anticuerpos en el hato y en los individuos del hato, incrementarían la susceptibilidad a nuevos desafíos de campo. En este contexto, mantener programas de vacunación con coberturas mayores al $80 \%$ ayudaría a reducir los brotes epizoóticos de la enfermedad en estos animales. ${ }^{4}$ En la actualidad se ha participado en investigaciones con el fin de caracterizar ciertos componentes anatómicos, fisiológicos e inmunológicos del tracto intestinal de las alpacas, producto de estas investigaciones, se han determinado la dinámica linfoide en el tracto intestinal de crías de alpacas ${ }^{17-19}$, la cinética de expresión de IgA en crías de alpacas ${ }^{20-22}$, expresión de péptidos antimicrobianos en mucosa intestinal de crías de alpacas. ${ }^{23,24}$

Con ese fin, el presente estudio busca una respuesta de protección inmune a las crías provenientes de las madres a través del calostro y evaluar el efecto de la administración de toxoides derivados de Clostridium perfringens tipo A y cómo responden los individuos tratados a un reto experimental con la cepa causante de la enterotoxemia, determinando la presencia o no de agentes que producen diarreas en crías de alpacas, esto a fin de brindar una visión de una nueva estrategia de control de la enfermedad con el propósito de reducir la mortalidad de las crías de alpacas por enterotoxemia.

\section{Materiales y métodos}

Lugar de estudio. Se realizó en el rebaño del Instituto Veterinario de Investigaciones Tropicales y de Altura (IVITA) Marangani y Laboratorios de Microbiología del IVITA Marangani, de la Facultad de Medicina Veterinaria de la Universidad Nacional
Mayor de San Marcos durante la época de parición del 2015, localizado en el distrito de Maranganí departamento de Cusco a $15^{\circ}$ latitud Sur y $70^{\circ}$ longitud Oeste, a una altura de 3600-4200 msnm. Presenta dos periodos estacionales marcados, uno seco (Abril a Noviembre) y otro de lluvia (Diciembre a Marzo), el promedio de precipitación pluvial anual es de $953 \mathrm{~mm}$, presenta temperaturas máximas que varían entre 13 y $19{ }^{\circ} \mathrm{C}$ y temperaturas mínimas que varían entre -5 y $1.9^{\circ} \mathrm{C}$ (SENAMHI 2014).

Animales. Se utilizaron 80 madres gestantes de alpacas y sus respectivas crías de la parición 2015 del rebaño de La CICAS La Raya-UNSAAC, cuya alimentación es a base de pasto natural de manera extensiva de las 8 am hasta a las 5 pm con rotación periódica de la zona de pastoreo, los animales fueron divididos en 4 grupos de 20 animales cada uno, en forma aleatoria de la siguiente manera:

\footnotetext{
1). Madres vacunadas y sus crías vacunadas. MV-CV

2). Madres no vacunadas y sus crías vacunadas. MnV-CV

3). Madres vacunadas y sus crías no vacunadas. $\mathrm{MV}-\mathrm{CnV}$

4). Madres no vacunadas y sus crías no vacunadas. $\mathrm{MnV}-\mathrm{CnV}$
}

A las madres de los grupos 1 y 2 se aplicaron $2 \mathrm{~mL}$ de toxoide al noveno y décimo mes de gestación.

A las crías de los grupos 1 y 3 se le aplicó $2 \mathrm{~mL}$ del toxoide a los 10 días de nacida.

Preparación del toxoide de cepas de campo de Clostridium perfringens Tipo A. De 10 casos clínicos de enterotoxemia de crías de alpacas muertas se aislaron cepas de Clostridium perfringens del contenido intestinal, los Clostridios, inicialmente se cultivaron en caldo tioglicolato (Merck) durante 48 h a $37{ }^{\circ} \mathrm{C}$ en jarras de anaerobiosis con sistemas de bolsas de generación de gas GasPak EZ, de las cuales se tomó una alícuota y se cultivó en agar cerebro corazón adicionando $10 \%$ de sangre de ovino desfibrinada en las mismas condiciones de anaerobiosis, el resultado de este sembrado se cultivó en el medio 
de caldo carne (Cooked Meat Broth-Sigma Aldrich) para conservar las cepas en estado vegetativo. Los aislados fueron sembrados en agar yema de huevo durante 24 a 48 h a $37{ }^{\circ} \mathrm{C}$ en anaerobiosis. En los medios de cultivo se verificaron las reacciones de las bacterias encontradas y fueron reactivadas en caldo tioglicolato. ${ }^{6}$ De las colonias obtenidas en los cultivos se realizaron tinciones de Gram para verificar las características morfológicas de las bacterias encontradas además de pruebas bioquímicas para su tipificación.

Del cultivo reactivado en agar sangre se tomó una ansada para sembrarlo en caldo tioglicolato utilizado para la preparación del toxoide que fue inactivado con formaldehido al $1 \%$ a las 24 h de incubación, se dispenso en frascos de $100 \mathrm{~mL}$, se guardaron refrigerados a $4{ }^{\circ} \mathrm{C}$ hasta la aplicación a madres y crías, para verificar la protección del toxoide en crías de alpacas contra enterotoxemia.

Comparación de la presencia de Clostridium perfringens en animales vacunados con los no vacunados y diagnóstico de la enfermedad. Se tomaron muestras de heces diarreicas de animales enfermos por enterotoxemia en horas de la mañana antes de salir al pastoreo y se analizó de acuerdo al protocolo para detección de $C$. perfringens tipo $\mathrm{A}^{6}$ y de otras bacterias y parásitos que causan diarreas en crías de alpacas. El diagnóstico se realizó mediante la presentación de signos en los animales enfermos como diarreas, depresión, postración y análisis de muestras en el laboratorio.

Determinación de la protección de las crías. Se registraron los datos de mortalidad y morbilidad de cada grupo, así como la asociación entre el control y los animales tratados.

Generar un modelo de control para reducir la mortalidad de crías por enterotoxemia en alpacas. Mediante los resultados entre grupos por la presencia o 59 no de agentes causantes de diarreas se creara un protocolo de vacunación contra la enterotoxemia.

\section{Resultados}

Preparación del toxoide de cepas de campo de Clostridium perfringens Tipo A. A la observación de Clostridium perfringens del contenido intestinal de crías de alpacas de los 10 casos clínicos de enterotoxemia sembrados en los medios diferenciales de agar sangre, agar yema de huevo, caldos de tioglicolato y carne se observan reacciones como doble hemolisis (alfa y beta hemólisis), lecitinasa (halo opaco alrededor de la colonia) y gases positivo respectivamente.

A La morfología microscópica de los cultivos obtenidos en caldo tioglicolato, agar sangre, caldo carne y agar yema de huevo de los 10 casos clínicos de enterotoxemia de las crías de alpacas fueron positivos a Clostridium perfringens, observándose bacilos Gram positivos, anchos y cortos con "apariencia de ladrillo".

A partir de los cultivos puros se realizó la caracterización bioquímica cuyos resultados son: lecitinasa + , catalasa -, hidrólisis gelatina,$+ \mathrm{H}_{2} \mathrm{~S}+$, fermentación glucosa + , lipasa -, indol -, crecimiento aerobio -, urea -, nitratos + , movilidad -, esculina - y gas + . Por las características morfológicas de las colonias de los medios de cultivos diferenciados, las tinciones de Gram y las reacciones bioquímicas se identificaron cultivos puros de Clostridium perfringens tipo A productores de toxina alfa y se preparó un toxoide a base de estas cepas.

Comparación de la presencia de Clostridium perfringens en animales vacunados con los no vacunados y diagnóstico de la enfermedad. En tres crías de los grupos 1 y 2 solo presentaron signos de diarreas verdosas oscuras, a los análisis de heces observó 
Eimeria $\mathrm{sp}$, siendo la presencia de las Eimeria $\mathrm{sp.}$ en el grupo 2 a los 15 días y en grupo 1 a los 18 días de nacidas, en el grupo 3, las crías presentaron signos de decaimiento y diarreas, aislándose de las heces diarreicas verdosas y amarillentas a partir de los nueve días Escherichia coli y a partir del día 15 de nacidas Eimeria sp. En el grupo 4 seis crías presentaron decaimiento dolor y diarreas cremosas amarillentas y grises desde los siete días de nacidas aislándose Clostridium perfringens y E. coli y a partir del día 15 se observó Eimeria sp.

En la tabla 1 se puede observar el resumen de la presentación por grupos de los signos, muestras, tiempo de vida de la toma de muestra con los resultados y el diagnostico de los agentes encontrados.
Determinación de la protección de las crías. No se presentaron casos de mortalidad, los porcentajes de morbilidad fueron $15 \%, 15 \%, 25 \%$ y $30 \%$ para los grupos 1, 2, 3, y 4 respectivamente. Resultado que confirma que la administración del toxoide preparado a base de cepas de Clostridium perfringens tipo A indujo mayor protección en los grupos 1 y 2 que en los grupos 3 y 4 .

Modelo de control para reducir la mortalidad de crías por enterotoxemia en alpacas. Un protocolo sugerido de acuerdo a los resultados es una vacunación masal a todas las hembras preñadas y a las crías del hato de alpacas de acuerdo a lo realizado en el protocolo del grupo 1 .

Tabla 1 Comparación de signos, muestras, tiempo de muestras agentes encontradas en los grupos de madres-crías vacunadas y no vacunadas

\begin{tabular}{|c|c|c|c|c|c|}
\hline $\begin{array}{c}\text { Grupo }(\mathbf{N} \\
20)\end{array}$ & $\mathbf{n}$ & Signos & Muestras Heces y color & Obtención muestras Días & Resultados Dx \\
\hline 1. MV-CV & 3 & Diarreas & Verdosas oscuras & 18 a 25 & Eimeria sp. \\
\hline 2. $\mathrm{MnV}-\mathrm{CV}$ & 3 & Diarreas & Verdosas oscuras & 15 a 25 & Eimeria sp. \\
\hline 3. MV-CnV & 5 & Decaimiento y Diarreas & Verdosas y Amarillentas & 09 a 25 & E. coli, Eimeria sp. \\
\hline 4. $\mathrm{MnV}-\mathrm{CnV}$ & 6 & Decaimiento, Dolor y diarreas & $\begin{array}{c}\text { Cremosas, amarillentas y } \\
\text { grises }\end{array}$ & 07 a 25 & $\begin{array}{c}\text { C. Perfringens, E. coli } \mathrm{e} \\
\text { Eimeria } \mathrm{sp} .\end{array}$ \\
\hline
\end{tabular}

\section{Discusión}

La enterotoxemia es una enfermedad infecciosa aguda que afecta a las crías de alpacas dentro el primer mes de vida. La enfermedad es caracterizada por presentar un cuadro de toxemia generalizado en el animal debido a la acción de las toxinas del Clostridium perfringens tipo $\mathrm{A}^{14}$, la presentación clínica de la enfermedad corresponde a una toxemia fatal producto de una enteritis hemorrágica o necrótica selectiva del yeyuno e íleon. ${ }^{10,1}$

El diagnóstico de la enfermedad en crías de alpacas se efectúa ocasionalmente en situaciones de campo a la presentación de brotes. La confirmación de laboratorio se lleva a cabo por procedimientos directos, como recuento en cultivo o amplificación del gen de la enterotoxina por PCR y por métodos indirectos de identificación de la toxina en heces mediante ELISA o aglutinación pasiva reversa por látex (APRL). Las técnicas de PCR se muestran muy útiles, pero pueden quedar fuera de la rutina de muchos laboratorios. Los métodos de detección antigénica pueden resultar más sencillos. Si bien se dispone de información sobre el empleo de métodos comerciales para la detección de otras toxinas de Clostridium los datos relativos al rendimiento de los kits destinados a la investigación de la toxina de $C$. perfringens son escasos. ${ }^{6}$ 
Durante las campañas de parición de 2005 a 2007 en ganaderías alpaqueras de los Andes peruanos, se logró aislar 234 cepas de $C$. perfringens, basados en la morfología de colonia, patrones hemolíticos, coloración Gram y pruebas de catalasa negativa. Estas cepas fueron sometidas a caracterizaciones bioquímicas, demostrando que 219 cepas mostraron actividad enzimática (positivos a la reacción de Nagler) asociada a producción de toxina alfa, y el $87 \%$ (190/219) redujo el sulfito en Agar Triptosa Sulfito Cicloserina (TSC) enriquecido con yema de huevo, característica del $C$. perfringens. La gran mayoría de estas cepas produce, in vitro, hemolisis incompleta y reacción positiva a Nagler, demostrando claramente que la mayoría de estos aislados expresan actividad fosfolipasa, inherente a la expresión de la toxina $\alpha$ proveniente de Clostridium perfringens tipo A. ${ }^{25}$

Análisis moleculares realizados en colonias de cada aislado positivo, revelaron que 223/224 (99.6\%) de las cepas contenían únicamente el gen cpa codificante de la toxina $\alpha$ (C. perfringens genotipo A) y solamente una de estas cepas $(0.4 \%)$ fue positiva a la presencia de ambos genes ( $c p a$ y $c p b$ ) codificantes de las toxinas $\alpha$ y $\beta$, que corresponden al genotipo C. En un análisis paralelo en todas estas cepas se detectaron genes de toxinas secundarias (subtipificación), indicando que el $91 \%$ de los aislados pertenecientes al genotipo A no tenían genes codificantes de la cpe ni el gen variante $c p b 2$. Las 19 restantes $(8.5 \%)$ mostraron, además, la presencia del gen $c p b 2$ pero negativos al gen cpe. La cepa perteneciente al genotipo $\mathrm{C}$ fue la única cepa positiva al gen cpe pero negativo para el $c p b 2$. Estos resultados evidencian que las exotoxinas secretadas, y no las endotoxinas (cpe), serían los probables factores de virulencia clostridiales productores de fatalidades en la enterotoxemia de la alpaca. ${ }^{25}$

61
En base a esta información los resultados mostraron que los microorganismos recuperados a partir de las muestras de intestino obtenidas de crías de alpacas con signos característicos de enterotoxemia como cólicos, distención abdominal, diarrea, y analizados empleando medios de cultivo enriquecidos y diferenciales fueron positivos para $C$. perfringens, llegando a obtenerse aislamientos productores de toxinas alfa confirmados en su género y especie por morfología macroscópica observándose al crecer en agar bajo condiciones anaerobias colonias características con forma de huevo frito, de aspecto rugoso y de color blanco, la morfología microscópica mostro bacilos Gram (+) con espora ovalada subterminal para los aislamientos. Las colonias obtenidas en agar sangre mostraron hemolisis positiva beta y alfa lo que indica una hemolisis completa de los glóbulos rojos producida por la toxina alfa $(\alpha)$ y en agar yema de huevo se observó alrededor de las colonias una zona opalescente característica donde la acción de la toxina hace que el lípido $P$ de la yema de huevo se descomponga en un componente insoluble y la lecitina en fosforilcolina y diglicerido.

Esta descomposición de la lecitina hace que se observe este color claro ya que la lecitina actúa como un agente estabilizante en la emulsión proteína y grasa la cual se rompe por acción de la toxina alfa ( $\alpha$ ) La caracterización bioquímica de 12 cepas de $C$ perfringens ha demostrado variabilidad en la actividad fosfolipídica, observándose que la mitad de estas cepas son productoras de alta actividad fosfolipasa asociada con una buena actividad de lecitinasas. ${ }^{6}$ La producción de las toxinas posiblemente este también asociada a la composición del medio de cultivo donde la carne incluida en el medio aporta proteínas libres solubles en agua que induce al microorganismo a producir las toxinas. La producción 
de gas $\left(\mathrm{H}_{2}\right.$ y $\left.\mathrm{CO}_{2}\right)$ en medio de carne fue un indicio de su metabolismo anaerobio.

Los resultados confirmaron que la administración del toxoide preparado a base de cepas de Clostridium perfringens tipo $\mathrm{A}$, probablemente indujo protección en los animales de los grupos de alpacas madres crías ya que aparentemente neutralizó la toxina alfa $(\alpha)$ que produciría el Clostridium perfringens tipo A en crías de alpacas con enterotoxemia.

El estudio permite obtener cepas de $C$. perfringens tipo A, a partir de muestras de animales con enterotoxemia que al ser bien caracterizadas, pueden emplearse para la elaboración de toxoides que inducen protección contra las enfermedades producidas por este microorganismo a nivel de campo y que el toxoide sería específico para la zona de donde se realizó el aislamiento.

Los resultados obtenidos llevan a concluir que mediante aislamientos de cepas de campo se puede obtener un toxoide para inducir la producción de defensas (IgG) como como una alternativa para reducir la enterotoxemia en crías de alpacas.

\section{Conflictos de intereses}

El presente trabajo ha cumplido con normas éticas en el manejo de los animales y no genera conflictos de interés.

\section{Agradecimientos}

A la Universidad Nacional Mayor de San Marcos por el financiamiento del estudio dentro del marco del proyecto Con Asignación a la Investigación y Con Incentivo al Investigador, código 150801111.

\section{Literatura citada}

1. Ameghino E, De Martini J. Mortalidad en Crías de Alpacas. Centro de Investigación Instituto Veterinario de Investigaciones Tropicales y de Altura. Lima Perú; 1991. p. 128.

2. Franco E, Pezo D, García W. Gestión de centros de producción de reproductores de alpacas y llamas Pub. Tec. $\mathrm{N}^{\mathrm{o}}$ 37. Universidad Nacional Mayor de San Marcos. Facultad de Medicina Veterinaria. Instituto Veterinario de Investigaciones Tropicales y de Altura; 1998. p. 1-33.

3. Pezo D, García W, Franco E. Ensayo del control de la enteritis neonatal en crías de alpacas y llamas XXI Reunión Científica Anual APPA. FMVZ - UNA-P. Puno Perú; 1998. p. 248-9.

4. Yaya K, Rosadio R. Ensayo de tres programas de vacunación anticlostridial en alpacas. Rev Investig Vet Perú 2005; 16(1):49-55. http://dx.doi. org/10.15381/rivep.v16i1.1534

5. Rosadio R, Yaya K, Véliz A, Quispe T. Efecto protector de una vacuna polivalente anticlostridial sobre la mortalidad neonatal en alpacas. Rev Investig Vet Perú 2012;23(3):299-306.

6. Pérez-Janampa DR. Genotipificación y subtipificación de Clostridium perfringens aisladas de crías de alpacas muertas por enterotoxemia. [Tesis de Licenciatura]. Universidad Nacional Mayor de San Marcos. Facultad de Medicina Veterinaria. Lima. Perú; 2006. p. 91.

7. Tuovinen E, keto J, Nikkilä J, Mättö J, Lähteenmäki K. Cytokine response of human mononuclear cells induced by intestinal Clostridium species. Anaerob 2013;19(1):70-6. http://dx.doi.org /10.1016j.anaerobe.2012.11.002

8. Ma Y, Chen Q, Ross AC. Retinoic acid polyriboinosinic: Polyribocytidylic acid stimulate robust anti-tetanus antibody production while dif- 
ferentially regulating type 1/type 2 cytokines and lymphocytes populations. J Immunol 2005; 174(12): 7961-9. https://doi.org/10.4049/ jimmunol.174.12.7961

9. Lázaro R, Manchego A, Pezo D, More J, Castro $\mathrm{G}$, Siuce J, et al. Efecto de antígenos de Clostridium perfringens y ácido retinoico sobre la expresión de IgA en la mucosa intestinal de crías de alpacas (Vicugna pacos). Rev Investig Vet Perú 2015;26(4): 689-97.

10. Moro M. Enfermedades infecciosas de las alpacas. Revista de Camélidos Sudamericanos 1987; 4: 7-31.

11.Prehn S, Sáez S, Arriagada M. Estudios microbiológicos y clínicos de enterotoxemia por Clostridium perfringens en camélidos chilenos. II Congreso Mundial de Camélidos Sudamericanos. Cuzco, Perú; 1999. p. 140.

12.Fowler ME. Medicine and surgery of South American Camelids: Llama, Alpaca, Vicuna, Guanaco. Iowa State University Press, AMES. $2^{\text {nd }}$ ed.1999. p. 166-9.

13. Rosadio R, Ameghino E. Enfermedades de los ovinos en el Perú. Publ Téc. Facultad de Medicina Veterinaria-Universidad Nacional Mayor de San Marcos; 1999; 40. p. 81.

14.Ramírez A. Alpaca Clostridium perfringens type A enterotoxemia: purification and assays of the enterotoxin. [Tesis Doctoral]. Colorado State University; 1987. p. 201.

15.Bravo PW, Garnica J, Fowler ME. Immunoglobulin $G$ concentrations in periparturient llamas, alpacas and their crías. Small Rumin Res 1997; 26(1-2):145-9. https://doi.org/10.1016/ S0921-4488(96)00965-0

16.Outteridge P. Veterinary immunology. Academic Press. London, UK; 1985. p. 280.
17.Roca V, Manchego A, Sandoval N, Chiok KL, Rivera H. Caracterización histológica y dinámica linfoide de las placas de Peyer en crías de alpacas durante los primeros 45 días de vida. Rev Investig Vet Perú 2011;25:341-9.

18. Herrera AR. Detección de citoquinas inductora y efectora para la diferenciación y función de linfocitos colaboradores 17 (Th17) en mucosa intestinal de crías de alpaca (Vicugna pacos). [Tesis de Maestría]. Universidad Nacional Mayor de San Marcos; 2012. p. 98.

19. Tambillo L, Manchego A, Kim-Lam CC, Sandoval N, More J, Rivera H. Evaluación in vitro de la respuesta leucocitaria de alpacas (Vicugna pacos) en presencia de antígenos clostridiales. Rev Inv Vet Perú 2013;24(4):510-23.

20.Dionisio J, Manchego A, Kim-Lam CC, Sandoval N, More J, Pezo D, et al. Cinética de expresión de inmunoglobulina A en el epitelio intestinal de crías de alpacas (Vicugna pacos). Rev Inv Vet Perú 2014; 25(2):151-61.

21.Chiok K. Expresión de citoquinas de la respuesta Th1 (IFN gamma e IL-2) y Th2 (IL-4 e IL-10) en mucosa intestinal de crías de alpaca (Vicugna pa$\cos$ ) sanas y con enteropatía. [Tesis de Maestría]. Universidad Nacional Mayor de San Marcos. Lima; 2012. p. 126.

22.Bardales C, Manchego A, Kim-Lam CC, Sandoval N, More J, Pezo D, et al. Cinética de expresión del factor de necrosis tumoral alfa (TNF- $\alpha)$ e interleucina 1 alfa (IL-1 $\alpha$ ) en mucosa intestinal de crías de alpaca (Vicugna pacos) sanas y con enteropatía. Rev Inv Vet Perú 2013;24(3):381-9.

23.More J, Manchego A, Sandoval N, Ramírez M, Pezo D, Chiok KL, et al. Detección genómica y expresión de péptidos antimicrobianos ( $\alpha$-y $\beta$ defensinas) en mucosa intestinal de crias de alpa- 
ca (Vicugna pacos). Rev Inv Vet Perú 2011;22 (4):324-35.

24. Siuce J, Manchego A, Sandoval N, More J, KimLam CC, Pezo D, et al. Expresión de defensinas en yeyuno de crías de alpacas (Vicugna pacos) con enteropatías. Rev Inv Vet Perú 2015; 26(2): 317-27.
25.Rosadio R, Maturrano L, Pérez D, Castillo H, Véliz A, Luna L, et al. Avances en el estudio de la patogénesis y prevención de la enterotoxemia de las alpacas. Rev Inv Vet Perú 2012;23 (3): 251-60. 